\title{
The American Journal of Sports Medicine
}

http://ajs.sagepub.com/

\section{Epidemiological Analysis of Factors Influencing an Episode of Exertional Rhabdomyolysis in High} School Students

Hsingwen Lin, Weichu Chie and Huangchun Lien

Am J Sports Med 2006 34: 481

DOI: $10.1177 / 0363546505281243$

The online version of this article can be found at:

http://ajs.sagepub.com/content/34/3/481

\author{
Published by: \\ (\$) SAGE \\ http://www.sagepublications.com \\ On behalf of:

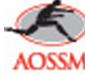 \\ American Orthopaedic Society for Sports Medicine
}

Additional services and information for The American Journal of Sports Medicine can be found at:

Email Alerts: http://ajs.sagepub.com/cgi/alerts

Subscriptions: http://ajs.sagepub.com/subscriptions

Reprints: http://www.sagepub.com/journalsReprints.nav

Permissions: http://www.sagepub.com/journalsPermissions.nav 


\title{
Epidemiological Analysis of Factors Influencing an Episode of Exertional Rhabdomyolysis in High School Students
}

\author{
Hsingwen Lin, ${ }^{\star} \mathrm{MD}, \mathrm{MPH}$, Weichu Chie, ${ }^{\dagger} \mathrm{MD}, \mathrm{PhD}$, and Huangchun Lien, ${ }^{\ddagger} \mathrm{MD}$ \\ From the ${ }^{*}$ Department of Family Medicine, Taipei County Hospital, Taipei, Taiwan, the ${ }^{\dagger}$ College \\ of Public Health, National Taiwan University, Taipei, Taiwan, and the ${ }^{\S}$ Department of Pathology, \\ National Taiwan University Hospital, National Taiwan University, Taipei, Taiwan
}

Background: An episode of rhabdomyolysis occurred after an endurance test in high school students in Taipei County in November 2003.

Purpose: To determine the incidence, outcome, and risk factors in an episode of exertional rhabdomyolysis in high school students.

Study Design: Descriptive epidemiology study.

Methods: We enrolled all 225 high school students who had performed an endurance test. Using data from retrospective questionnaires, we estimated the incidence and assessed risk factors of exertional rhabdomyolysis among these students. Multiple logistic regression was used to determine risk factors associated with exertional rhabdomyolysis.

Results: The completed questionnaire was returned by $70 \%$ (157 of 225) of the students. Of these, $43.3 \%$ (68 of 157 ) were identified as having exertional rhabdomyolysis, and the incidence was not statistically different between male and female students $(P=.49)$. Dark urine was noted in only $25 \%$ of the students. None of the students developed acute renal failure. The risk of exertional rhabdomyolysis was significantly higher in those students who had not exercised 1 day before the endurance test (odds ratio [OR], 6.10; 95\% confidence interval [Cl], 2.00-18.00) and those who had performed postexercise stretching of the legs (OR, $3.13 ; 95 \% \mathrm{Cl}, 1.28-7.69)$ or performed complete squats during the test $(\mathrm{OR}, 3.21 ; 95 \% \mathrm{Cl}, 1.12-10.00)$. There were no statistically significant differences in gender, body mass index, presence of flulike symptoms, previous exercise routine, and medication history between students with or without exertional rhabdomyolysis.

Conclusion: Our findings suggest that exertional rhabdomyolysis is not uncommon in strenuous eccentric exercise in both men and women, but the risk of developing acute renal failure is very low. Exercise 1 day before eccentric exercise was significantly associated with a reduced risk of exertional rhabdomyolysis, whereas postexercise stretching of the involved extremities might increase the risk.

Keywords: eccentric exercise; exertional rhabdomyolysis; creatine kinase; renal failure

Rhabdomyolysis is a potentially life-threatening syndrome resulting from the breakdown of skeletal muscle fibers, with leakage of muscle proteins such as creatine kinase (CK) and myoglobin into the blood. ${ }^{23}$ Since the original description of the syndrome of muscle pain, weakness, and brown urine by Meyer-Betz in $1910,{ }^{20}$ many case reports have linked rhabdomyolysis to strenuous activities, such

\footnotetext{
${ }^{\ddagger}$ Address correspondence to Huangchun Lien, MD, N. 7, Chun-Shan S. Road, Taipei, Taiwan 105 (e-mail: lienhc@ha.mc.ntu.edu.tw).

No potential conflict of interest declared.
}

The American Journal of Sports Medicine, Vol. 34 , No. 3 DOI: 10.1177/0363546505281243

(C) 2006 American Orthopaedic Society for Sports Medicine as basic military training, weight lifting, and marathon running; these conditions are collectively termed exerciseinduced rhabdomyolysis or exertional rhabdomyolysis (ER). ${ }^{9}$

Exercises that are biased toward eccentric contractions, in which the muscles are lengthening while trying to contract, are particularly damaging to muscle fibers partly because of the increased strain placed on muscle tissues. ${ }^{4,521}$ Exercises with repetitive and strenuous eccentric contractions have been reported to be related to rhabdomyolysis. ${ }^{3}$ The criteria for ER are generally accepted as a recent strenuous exercise event and serum CK levels more than 1000 IU/L, or 5 times the normal upper limit. ${ }^{8,11,14,17,34}$ Although many cases of ER have been reported in the literature, its incidence remains difficult to estimate and may be underestimated for 2 main reasons. First, these cases 
are mainly case reports that came to the attention of the medical community mainly because of myalgia, muscle tenderness, and dark urine, which are not always present in rhabdomyolysis. Second, serum CK levels, a widely used marker for the amount of muscle damage, are usually not available for the entire population during an episode of ER. Thus, cases of subclinical ER with abnormally high CK levels but no major symptoms are usually underdiagnosed and underestimated. Accordingly, the incidence, as well as factors influencing the onset, of ER remains largely unknown.

An endurance test is sometimes used to evaluate the physical fitness of students in Taiwan, and there was an episode of rhabdomyolysis after such a test in high school students in Taipei County in November 2003. The purpose of this study was to determine the incidence, risk factors, and outcome in an episode of ER in the high school students who had performed the endurance test, using data from retrospective questionnaires.

\section{METHODS}

\section{Background}

An episode of rhabdomyolysis occurred after an endurance test in high school students in November 2003. Initially, several students developed dark urine 1 day after the endurance test and were diagnosed as having ER. The teachers therefore asked all the students who had participated in the endurance test to go to the hospital to be screened for ER. All 225 students involved had their serum CK levels checked.

\section{Study Population and Design}

The study population included all 225 students who had performed the endurance test, which consisted of 1 minute of curl-ups, 1 minute of push-ups, and 8 minutes of squatstand repetitions, with the score for the test depending only on the number of squat-stand repetitions. Retrospective questionnaires were administered to students 1 month after the endurance test. All the students were informed on the questionnaire about the purpose and nature of the study and about having a free choice to complete and return the questionnaire.

The questions on potential risk factors associated with ER were designed by analyzing open questions in a pilot study of 15 students with ER in this episode and by searching the English-language medical literature in PubMed from 1960 to 2003. The questionnaires contained questions about body weight and height, gender, number of squatstand repetitions, whether the squats were complete or partial, the presence of myalgia, muscle tenderness, muscle weakness, general weakness, muscle swelling, dark urine, serum CK levels at admission, adequate hydration before and after the endurance test (defined as drinking more than $2 \mathrm{~L}$ of water per day), postexercise stretching, alcohol or drug use, smoking, presence of flulike symptoms, history of regular exercise (defined as three 30-minute periods of exercise per week), exercise (defined as at least 30 minutes of exercise) 1 day before the endurance test, heat shock history, medical and medication history, and whether there was a family history of myopathy and rhabdomyolysis.

\section{Definition and Confirmation of Cases}

Serum CK levels at admission were used to evaluate the presence of rhabdomyolysis. Although there are no standard CK values that establish the diagnosis of rhabdomyolysis, it is generally accepted that an acute increase in serum CK levels to more than $1000 \mathrm{IU} / \mathrm{L}$, or 5 times the normal upper limit, indicates rhabdomyolysis. ${ }^{8,11,14,17,34}$ In this study, students with ER were defined as those with CK levels higher than 1000 IU/L.

\section{Statistical Analysis}

Statistical analysis was performed using SPSS software, version 10.0 (SPSS Science Inc, Chicago, Ill). The unpaired Student $t$ test was used to compare continuous variables, and the $\chi^{2}$ test was used for categorical variables. Risk factors found to show a significant association with ER by univariate analysis were further analyzed using the multivariate logistic regression model. In both, a 2 -sided test with $P<.05$ was judged to be significant.

\section{RESULTS}

We received complete data for the questionnaire from 157 students, the response rate being $70 \%$ (157 of 225). Of these 157 students, $43.3 \%$ (68 of 157) had serum CK levels $\geq 1000 \mathrm{IU} / \mathrm{L}$ and were diagnosed as having ER. The demographic characteristics of the 157 students are summarized in Table 1. Gender, body weight, height, body mass index, and the number of squat-stand repetitions showed no statistically significant difference between students with or without ER.

Table 2 shows the clinical symptoms and signs in students with or without ER. Of these, dark urine, myalgia, and muscle swelling were significantly more frequent in students with ER. Dark urine was not seen in any students without ER and was seen in only $25 \%$ of cases with ER. There was no significant difference in the frequency of muscle weakness and general weakness between students with or without ER.

The incidence of ER in male and female students was $45.3 \%$ (48 of 106) and 39.2\% (20 of 51), respectively, and the distribution of their CK values is summarized in Table 3. The mean CK levels in male and female students (41563 \pm 53226 vs $41400 \pm 32419$ IU/L) were not significantly different $(P=.99)$. Four male students had CK levels of more than $200000 \mathrm{IU} / \mathrm{L}$, the highest being $260000 \mathrm{IU} / \mathrm{L}$.

Analyses of potential risk factors associated with ER are summarized in Table 4. Univariate analysis showed that ER was significantly associated with no exercise 1 day before the endurance test $(P<.001)$, inadequate hydration after the test $(P=.03)$, postexercise stretching $(P=.002)$, and the performance of complete squats $(P=.03)$. Multivariate 
TABLE 1

Characteristics of Students Who Responded to the Questionnaire $(\mathrm{n}=157)$

\begin{tabular}{lcc}
\hline Characteristics & Cases, $\mathrm{n}=68$ & All Students, $\mathrm{n}=157$ \\
\hline Gender & & \\
$\quad$ Male & 48 & 106 \\
$\quad$ Female & 20 & 51 \\
Body weight, kg, & $60.96(10.19)$ & $62.15(10.92)$ \\
$\quad$ mean (SD) & & \\
Height, cm, mean (SD) & $168.28(8.22)$ & $168.23(8.12)$ \\
Body mass index, & $21.47(2.43)$ & $21.88(3.07)$ \\
$\quad$ kg/m, mean (SD) & & \\
Number of squat-stand & $200.25(41.90)$ & $198.68(40.37)$ \\
$\quad$ repetitions, mean (SD) & & \\
\hline
\end{tabular}

TABLE 2

Symptoms and Signs of Students With or Without Exertional Rhabdomyolysis

\begin{tabular}{|c|c|c|c|c|c|}
\hline \multirow[b]{3}{*}{ Symptoms and Signs } & \multicolumn{4}{|c|}{ Exertional Rhabdomyolysis } & \multirow{3}{*}{$P$} \\
\hline & \multicolumn{2}{|c|}{ Yes, $\mathrm{n}=68$} & \multicolumn{2}{|c|}{ No, $\mathrm{n}=89$} & \\
\hline & $\mathrm{n}$ & $\%$ & $\mathrm{n}$ & $\%$ & \\
\hline Dark urine & 17 & 25 & 0 & 0 & $<.001$ \\
\hline Myalgia & 44 & 65 & 40 & 45 & .02 \\
\hline Muscle weakness & 11 & 16 & 6 & 7 & .07 \\
\hline General weakness & 3 & 4 & 0 & 0 & .08 \\
\hline Muscle swelling & 9 & 13 & 2 & 2 & .01 \\
\hline
\end{tabular}

analysis of these 4 variables showed that no exercise 1 day before the endurance test (odds ratio [OR], 6.10; 95\% confidence interval [CI], 2.00-18.00), postexercise stretching (OR, $3.13 ; 95 \% \mathrm{CI}, 1.28-7.69)$, and the performance of complete squats (OR, 3.21; 95\% CI, 1.12-10.00) were risk factors statistically associated with ER. Exercise routine, adequate hydration before and after the test, and flulike symptoms were not significantly associated with ER. All 157 students stated that they had no history of glucose 6-phosphate dehydrogenase deficiency, hypothyroidism, hereditary myopathy, or rhabdomyolysis and that they were not taking antidepressants, antifungal agents, antibiotics, antihistamines, or narcotic drugs at the time of the endurance test. One student was a cigarette smoker but was not diagnosed as having ER. None of the 157 students, even the 4 students with CK levels above $200000 \mathrm{IU} / \mathrm{L}$, were told by doctors or paramedics that they had acute renal failure (ARF).

\section{DISCUSSION}

The pathogenesis of ER is not clear, although mechanical stress of skeletal muscle has been suggested to play a role. ${ }^{3}$ Muscle damage is induced more readily by eccentric contraction, in which the muscles are lengthening while trying to contract, perhaps because of increased strain on the
TABLE 3

Distribution of Creatine Kinase Levels in Male and Female Students With Exertional Rhabdomyolysis

\begin{tabular}{|c|c|c|c|c|c|}
\hline \multirow{2}{*}{$\begin{array}{l}\text { Creatine Kinase } \\
\text { Levels, IU/L }\end{array}$} & \multicolumn{2}{|c|}{ Male, $\mathrm{n}=48$} & \multicolumn{2}{|c|}{ Female, $\mathrm{n}=20$} & \multirow[t]{2}{*}{$P$} \\
\hline & $\mathrm{n}$ & $\%$ & $\mathrm{n}$ & $\%$ & \\
\hline $1000-9999$ & 14 & 30 & 3 & 15 & \\
\hline $10000-99999$ & 29 & 60 & 16 & 80 & \\
\hline 100 000-199999 & 1 & 2 & 1 & 5 & \\
\hline 200000 or more & 4 & 8 & 0 & 0 & \\
\hline Mean (SD) & \multicolumn{2}{|c|}{$41563(53226)$} & \multicolumn{2}{|c|}{41400 (32 419) } & .99 \\
\hline
\end{tabular}

muscle tissue. ${ }^{12}$ In 1960, Howenstine et al $^{13}$ reported 60 cases of rhabdomyolysis in Marine recruits in a single year after they performed numerous squat jumps, an exercise involving repetitive eccentric contractions. Other cases of ER occurring during military recruit training associated with eccentric exercise have been reported. ${ }^{6,7,29}$ This finding suggests that eccentric exercise might be associated with ER, but the incidence and risk factors remain largely unknown.

In the present study, all 225 students who had performed the endurance test were asked to go to the hospital for evaluation, and all were tested for serum CK levels. Of the symptoms and signs associated with rhabdomyolysis, dark urine has been regarded as the most specific. None of the 89 students without ER had dark urine, but only $25 \%$ (17 of 68) of those with ER showed this sign, suggesting that dark urine, although specific, is not a sensitive sign of ER. The remaining $75 \%$ (51 of 68) of ER cases might represent a subclinical form of ER, which would result in an underestimation of the incidence of ER if the serum CK levels were not available. The incidence of ER in the episode $(43 \%$; 68 of 157$)$ is very close to the value of $39.2 \%$ reported by Olerud et $\mathrm{al}^{22}$ in a prospective study examining serum myoglobin and CK, lactate dehydrogenase, and glutamic-oxaloacetic transaminase levels in 337 volunteer military recruits during training that would be expected to include some repetitive eccentric exercise. Taken together, these 2 studies suggest that ER is not uncommon in exercise involving repetitive eccentric exercise, irrespective of the sample population.

Although rhabdomyolysis associated with septic shock, electrolyte disturbances, or drug or alcohol abuse appears to occur at the same frequency in men and women, ER is rarely reported in women. ${ }^{16}$ Data from previous studies, summarized by Shumate et al, ${ }^{27}$ have suggested a lack of a CK response to moderately strenuous exercise in women but a rapid increase in CK levels in men, suggesting that estrogens may be a CK-protective factor and may be responsible for the difference in CK levels seen in response to exercise between men and women. Contrary to previous conceptions, we noted that the incidence of ER in female students was not significantly different from that in male students $(39.2 \%$ vs $45.3 \% ; P=.49)$, suggesting that $\mathrm{ER}$ is not common not only in men but also in women. In fact, 
TABLE 4

Analysis of the Risk Factors of Exertional Rhabdomyolysis in the 157 Students Responding to the Questionnaire

\begin{tabular}{|c|c|c|c|c|c|c|}
\hline \multirow[b]{2}{*}{ Variable } & \multirow[b]{2}{*}{$\mathrm{n}$} & \multirow[b]{2}{*}{ Cases, $\mathrm{n}=68$} & \multirow[b]{2}{*}{ Attack Rate, $\%$} & \multirow{2}{*}{$\frac{\text { Univariate Analysis }}{P}$} & \multicolumn{2}{|l|}{ Multivariate Analysis } \\
\hline & & & & & $\begin{array}{c}\text { Odds Ratio } \\
\text { (95\% Confidence Interval) }\end{array}$ & $P$ \\
\hline Gender & & & & .49 & & \\
\hline Male & 106 & 48 & 45 & & & \\
\hline Female & 51 & 20 & 39 & & & \\
\hline Routine exercise & & & & .42 & & \\
\hline No & 65 & 31 & 48 & & & \\
\hline Yes & 92 & 37 & 40 & & & \\
\hline $\begin{array}{l}\text { Exercise }(\geq 30 \min ) 1 \text { day } \\
\text { before the endurance test }\end{array}$ & & & & $<.001^{a}$ & $6.10(2.00-18.00)$ & .001 \\
\hline No & 120 & 62 & 52 & & & \\
\hline Yes & 37 & 6 & 16 & & & \\
\hline $\begin{array}{l}\text { Adequate hydration }(>2 \mathrm{~L} / \mathrm{d} \\
\text { before the endurance test }\end{array}$ & & & & .86 & & \\
\hline No & 111 & 49 & 44 & & & \\
\hline Yes & 46 & 19 & 41 & & & \\
\hline $\begin{array}{l}\text { Adequate hydration }(>2 \mathrm{~L} / \mathrm{d} \\
\text { after the endurance test }\end{array}$ & & & & $.03^{a}$ & $2.36(1.02-5.49)$ & .44 \\
\hline No & 43 & 25 & 58 & & & \\
\hline Yes & 114 & 43 & 38 & & & \\
\hline $\begin{array}{l}\text { Postexercise stretching after the } \\
\text { endurance test }\end{array}$ & & & & $.002^{a}$ & $3.13(1.28-7.69)$ & .01 \\
\hline No & 65 & 18 & 28 & & & \\
\hline Yes & 92 & 50 & 54 & & & \\
\hline $\begin{array}{l}\text { Flulike symptoms during the } \\
\text { endurance test }\end{array}$ & & & & .58 & & \\
\hline No & 143 & 60 & 42 & & & \\
\hline Yes & 14 & 8 & 57 & & & \\
\hline Complete squats & & & & $.03^{a}$ & $3.21(1.12-10.00)$ & .03 \\
\hline No & 27 & 7 & 26 & & & \\
\hline Yes & 130 & 61 & 47 & & & \\
\hline
\end{tabular}

${ }^{a}$ Risk factors with 2 -sided $P<.05$ by univariate analysis were further analyzed using the multiple logistic regression model.

Sayers and Clarkson ${ }^{24}$ found that in 32 subjects who demonstrated profound weakness and strength loss after eccentric exercise, 24 were women. However, it must be noted that even though the mean CK levels in the present study were not significantly different in male and female students with ER, all 4 students with CK levels of more than $200000 \mathrm{IU} / \mathrm{L}$ were male. This finding is in agreement with the finding that the most profound swelling and prolonged strength loss responses after eccentric exercise have been in men. ${ }^{24,25}$ In addition, it is interesting and noteworthy that 1 of these 4 students had an uncle diagnosed as having polymyositis, a disease characterized by muscle damage with high CK levels.

Although it is currently not clear whether certain diseases, especially those associated with muscular damage, may predispose a person to ER, our preliminary finding suggests a potential link between severe ER and certain genetic traits. This report, therefore, suggests that, contrary to previous conceptions, women may be equally susceptible to ER, although men may have a higher risk of developing severe ER, and that perhaps some cases may be related to a potential genetic predisposition, rendering the muscles more susceptible to damage.

Training can reduce the damage that occurs in response to a given exercise task possibly because of the adaptation of the muscle to future damage. Contrary to this common conception, we found that an exercise routine did not reduce the incidence of ER $(P=.2)$. However, we did find that exercise for more than 30 minutes 1 day before the endurance test was significantly associated with a reduced risk of $\mathrm{ER}(\mathrm{OR}, 6.10 ; 95 \% \mathrm{CI}, 2.00-18.00)$, and this potential risk factor remained significantly associated with ER (OR, 8.79; 95\% CI, 1.93-39.81), even when the criterion for rhabdomyolysis was set as CK levels $\geq 10000$ IU/L. This observation is further supported by the observations of MacLeay et $\mathrm{al},{ }^{19}$ who, in an epidemiological analysis of factors influencing ER in thoroughbred horses, found that ER frequently developed in horses that had $\geq 1$ day of rest before 
exercise. They proposed that a period of rest may result in excitability when training is resumed. ${ }^{19}$ In fact, rhabdomyolysis in swine has been shown to be associated with stress, excitement, and high catecholamine concentrations. ${ }^{19}$ Furthermore, in Standardbred horses with ER, serum catecholamine levels increased, parallel with serum CK levels. ${ }^{30}$ Although the relationship between catecholamine levels and muscle damage is not clear and the pathogenesis of ER may be variable among species, the identification of this novel epidemiological factor as being significantly related to ER is extremely important not only for ER prevention but also for future studies on the mechanism.

Studies on military training-related ER have suggested, but not proved, that the amount and type of exercise might be related to differences in serum myoglobin values between platoons. ${ }^{22}$ In this study, we found that complete squats resulted in a significantly increased risk of ER compared with partial squats, supporting the theory that the type of exercise is related to the risk of ER. It also should be mentioned that eccentric exercise, even though it increases the risk of rhabdomyolysis (potential negative outcome), is a deliberate part of the training-hypertrophy response through muscle breakdown and damage (potential positive outcome). Based on this finding, we suggest that training courses or competitive exercises involving complete eccentric contraction should be designed with great caution.

Some people, including athletes and fitness trainers, believe that postexercise stretching might help relieve pressure and soreness of the extremities involved. In fact, muscular activity has been suggested as the best treatment for the sensation of pain and stiffness after unaccustomed exercise. ${ }^{2}$ However, when comparing students who did and did not perform stretching, we found that postexercise stretching was significantly associated with an increased risk of ER. It is noteworthy that 1 of the students who repeatedly stretched the legs after the test (postexercise stretching) to reduce soreness later had muscle atrophy of the bilateral quadriceps. However, we cannot rule out muscle tightness or other sensations in students with impending ER that made them want to stretch, rather than stretching in and of itself, as a cause of ER.

Although the mechanism underlying this risk factor is not clear, our preliminary findings suggest that postexercise stretching after strenuous eccentric exercise, which itself might already make the subject prone to ER, might increase the risk of ER and should be avoided.

Rhabdomyolysis is a reported complication of some viral infections. ${ }^{18,32}$ It is not known whether viral infection is related to ER and whether subjects with flulike symptoms might have an increased risk of ER. In this report, we did not find a significant increase in the risk of ER in students with flulike symptoms, suggesting that these symptoms might not increase the risk.

The clinical significance of rhabdomyolysis lies in its association with ARF. The incidence of ARF secondary to rhabdomyolysis from all causes is reported to range from $17 \%$ to $50 \% .^{1,8,15,31,33}$ In addition, a weak predictive correlation between CK levels and the development of ARF has been claimed, with levels higher than 16000 IU/L being more likely to be associated with $\mathrm{ARF}^{33}$ It is noteworthy that although the mean CK levels in male and female students ( $41563 \pm 53226$ vs $41400 \pm 32419$ IU/L) in the current study were comparable with those in male and female patients (44600 \pm 71553 vs $32830 \pm 46579$ IU/L) who had crush syndrome as a result of the Marmara earthquake in Turkey $^{26}$ and with those in patients (median CK, 20000 IU/L; range, 1040-351 540 IU/L) who had crush syndrome as a result of the Taiwan Chi-Chi earthquake, ${ }^{14}$ the outcome was significantly different between patients having rhabdomyolysis secondary to exertion versus crush syndrome. None of our 68 patients with ER developed ARF and needed dialysis support, despite extremely high CK levels (more than $200000 \mathrm{IU} / \mathrm{L}$ ) in 4 of the patients. In contrast, about $70 \%$ of those patients in the Marmara earthquake needed dialysis support, ${ }^{26}$ and $54.7 \%$ of those patients in the Chi-Chi earthquake, who were diagnosed as having rhabdomyolysis using the same criteria as in the current study, developed ARF. ${ }^{14}$ Consistent with our finding, Sinert et $\mathrm{al}^{28}$ found that none of the 35 patients with ER developed $\mathrm{ARF}$, despite having similar mean serum CK levels to those in patients with ARF in previous reports. ${ }^{1,10,16}$ Taken together, these 2 findings suggest a much lower incidence of ARF in ER than in other forms of rhabdomyolysis. The pathophysiology underlying this difference remains to be elucidated.

Although there are currently no strict criteria for what we label as ER, the laboratory confirmation of rhabdomyolysis is generally accepted as an acute increase in serum CK levels to more than $1000 \mathrm{IU} / \mathrm{L}$, or 5 times the normal upper limit. ${ }^{8,11,14,17,34}$ In this study, having dark urine (17 of 157; $11 \%$ ), the more serious clinical manifestation of rhabdomyolysis, was much more rare than having high CK levels (68 of $157 ; 43 \%$ ) or even simple muscle pain (84 of $157 ; 54 \%$ ) from exercise. This finding suggests that although high levels of CK do indicate some level of muscle damage, high levels of CK are not always accompanied by myoglobin release that may compromise renal function. The value of CK as an indicator of ER remains debatable.

In attempting to determine risk factors of ER using a retrospective questionnaire analysis, several limitations must be acknowledged. First, because the questionnaires were administered 1 month after the event, the retrospective nature of the survey may have resulted in faulty recollections, possibly biased by knowledge of whether the person had ER. However, it must be noted that certain symptoms, such as muscle weakness and general weakness, and potential risk factors, such as no exercise routine, inadequate hydration before or after the test, and the presence of flulike symptoms, which might be more likely to be biased toward being present in cases with ER, were found not to be significantly associated with ER. In contrast, no exercise 1 day before the test, which was thought to be less likely to increase the risk of ER, was found to be an independent risk factor for ER. This finding is strongly supportive of the associations of risk factors found in this study, despite potential information bias in the retrospective survey. Second, because this study design was based on retrospective questionnaire analysis, some of the laboratory data for the students, such as serum and urine myoglobin levels and serial data on serum 
CK levels, and the detail of treatments for these students were not available. Some students in the pilot study declined further review of their medical charts, so no review was performed for any of the students. Finally, because this study is a retrospective survey, some of the negative findings in the analysis of potential risk factors of ER may be because of the small sample size. Nevertheless, despite these limitations, this report provides novel and valuable information on the epidemiology of ER. Further prospective case control studies will be important in validating these findings.

In conclusion, our findings suggest that if serum CK levels are evaluated, ER is not an uncommon finding in strenuous eccentric exercise and that both women and men are equally susceptible, although severe ER tends to occur in men. Exercise 1 day before strenuous exercise is significantly associated with a reduced risk of ER, whereas postexercise stretching might increase the risk of ER, with the rare but severe sequela of muscle atrophy. Military training, physical training courses, or competitive exercises involving repetitions of eccentric contractions should be designed with care, and all persons involved in eccentric exercise should be aware of the potential sequelae and take preventive action, especially adequate exercise 1 day before the training course or exercise. Finally, although ER is common in eccentric exercise, the potential risk of ARF is very low.

\section{ACKNOWLEDGMENT}

We are grateful to all the students who completed and returned the questionnaires for their time and openness. This study was funded by the National Science Council (NSC) of Taiwan, Grant 93-2320-B-002-121.

\section{REFERENCES}

1. Akmal M, Valdin JR, McCarron MM, Massry SG. Rhabdomyolysis with and without acute renal failure in patients with phencyclidine intoxication. Am J Nephrol. 1981;1:91-96.

2. Armstrong RB. Mechanisms of exercise-induced delayed onset muscular soreness: a brief review. Med Sci Sports Exerc. 1984;16:529-538.

3. Armstrong RB. Muscle damage and endurance events. Sports Med. 1986;3:370-381.

4. Clarkson PM, Nosaka K, Braun B. Muscle function after exerciseinduced muscle damage and rapid adaptation. Med Sci Sports Exerc. 1992;24:512-520.

5. Clarkson PM, Sayers SP. Etiology of exercise-induced muscle damage. Can J Appl Physiol. 1999;24:234-248.

6. Demos MA, Gitin EL. Acute exertional rhabdomyolysis. Arch Intern Med. 1974;133:233-239.

7. Demos MA, Gitin EL, Kagen LJ. Exercise myoglobinemia and acute exertional rhabdomyolysis. Arch Intern Med. 1974;134:669-673.

8. Gabow PA, Kaehny WD, Kelleher SP. The spectrum of rhabdomyolysis. Medicine (Baltimore). 1982;61:141-152.

9. Greenberg J, Arneson L. Exertional rhabdomyolysis with myoglobinuria in a large group of military trainees. Neurology. 1967;17:216-222.
10. Grossman RA, Hamilton RW, Morse BM, Penn AS, Goldberg M. Nontraumatic rhabdomyolysis and acute renal failure. $N$ Engl $J$ Med. 1974;291:807-811.

11. Gunal Al, Celiker H, Dogukan A, et al. Early and vigorous fluid resuscitation prevents acute renal failure in the crush victims of catastrophic earthquakes. J Am Soc Nephrol. 2004;15:1862-1867.

12. Hamer R. When exercise goes awry: exertional rhabdomyolysis. South Med J. 1997;90:548-551.

13. Howenstine JA. Exertion-induced myoglobinuria and hemoglobinuria; simulation of acute glomerulonephritis. JAMA. 1960;173:493-499.

14. Hwang SJ, Shu KH, Lain JD, Yang WC. Renal replacement therapy at the time of the Taiwan Chi-Chi earthquake. Nephrol Dial Transplant. 2001;16 (suppl 5):78-82.

15. Kageyama Y. Rhabdomyolysis: clinical analysis of 20 patients. Nippon Jinzo Gakkai Shi. 1989;31:1099-1103.

16. Knochel JP. Catastrophic medical events with exhaustive exercise: "white collar rhabdomyolysis." Kidney Int. 1990;38:709-719.

17. Lane R, Phillips M. Rhabdomyolysis. BMJ. 2003;327:115-116.

18. Lee $N$, Hui $D, W u A$, et al. A major outbreak of severe acute respiratory syndrome in Hong Kong. N Engl J Med. 2003;348:1986-1994.

19. MacLeay JM, Sorum SA, Valberg SJ, Marsh WE, Sorum MD. Epidemiologic analysis of factors influencing exertional rhabdomyolysis in Thoroughbreds. Am J Vet Res. 1999;60:1562-1566.

20. Meyer-Betz F. Beobachtugen an einem eigenartigen mit muskellahmungen verbundenen Fall von Hamoglobinurie. Dtsch Arch Klin Med. 1910;101:85-127.

21. Nosaka K, Clarkson PM. Variability in serum creatine kinase response after eccentric exercise of the elbow flexors. Int J Sports Med. 1996; 17:120-127.

22. Olerud JE, Homer LD, Carroll HW. Incidence of acute exertional rhabdomyolysis: serum myoglobin and enzyme levels as indicators of muscle injury. Arch Intern Med. 1976;136:692-697.

23. Sauret JM, Marinides G, Wang GK. Rhabdomyolysis. Am Fam Physician. 2002;65:907-912.

24. Sayers SP, Clarkson PM. Force recovery after eccentric exercise in males and females. Eur J Appl Physiol. 2001;84:122-126.

25. Sayers SP, Clarkson PM, Rouzier PA, Kamen G. Adverse events associated with eccentric exercise protocols: six case studies. Med Sci Sports Exerc. 1999;31:1697-1702.

26. Sever MS, Erek E, Vanholder R, Lameire N. Effect of gender on various parameters of crush syndrome victims of the Marmara earthquake. J Nephrol. 2004;17:399-404.

27. Shumate JB, Brooke MH, Carroll JE, Davis JE. Increased serum creatine kinase after exercise: a sex-linked phenomenon. Neurology. 1979;29:902-904.

28. Sinert R, Kohl L, Rainone T, Scalea T. Exercise-induced rhabdomyolysis. Ann Emerg Med. 1994;23:1301-1306.

29. Smith RF. Exertional rhabdomyolysis in Naval Officer Candidates. Arch Intern Med. 1968;121:313-319.

30. Valberg S, Haggendal J, Lindholm A. Blood chemistry and skeletal muscle metabolic responses to exercise in horses with recurrent exertional rhabdomyolysis. Equine Vet J. 1993;25:17-22.

31. Vanholder R, Sever MS, De Smet M, Erek E, Lameire N. Intervention of the Renal Disaster Relief Task Force in the 1999 Marmara, Turkey earthquake. Kidney Int. 2001;59:783-791.

32. Wang JL, Wang JT, Yu CJ, et al. Rhabdomyolysis associated with probable SARS. Am J Med. 2003;115:421-422.

33. Ward MM. Factors predictive of acute renal failure in rhabdomyolysis. Arch Intern Med. 1988;148:1553-1557.

34. Welch RD, Todd K, Krause GS. Incidence of cocaine-associated rhabdomyolysis. Ann Emerg Med. 1991;20:154-157. 